\title{
OSA: A VANET Application focused in Energy Efficiency
}

\author{
Oscar Arley Orozco \\ I2T Research Group \\ Universidad Icesi \\ Cali, Colombia \\ oaorozco@icesi.edu.co
}

\author{
Gonzalo Llano Ramírez \\ I2T Research Group \\ Universidad Icesi \\ Cali, Colombia \\ gllano@icesi.edu.co
}

\begin{abstract}
With the growth in the number of vehicles moving through the streets, high vehicular flow has become a mobility and public health problem for governmental institutions and people since time of travel, gasoline consumption, and greenhouse gas [GHG] emissions have suffered an important increase. Hereby, policies and actions are required to reduce the impact of this increase. In this context, we have developed an application for the simulation of Vehicular Ad hoc Networks [VANET] using two software applications: SUMO and OMNeT++. The application we developed optimizes fuel usage and reduces GHG emissions; showing that vehicles following a preset speed (previously studied), gasoline consumption and GHG emissions present a considerable decrease compared to vehicles not running at that speed.
\end{abstract}

Keywords- $\mathrm{CO}_{2}$; Energy Efficiency; Gasoline consumption; Greenhouse gas; I2V; OSA; VANET.

\section{INTRODUCCIÓN}

El acelerado desarrollo de la sociedad actual ha impulsado en las personas tendencias y comportamientos que antes se consideraban como poco probables. En particular, la obtención de un vehículo o motocicleta dejó de ser un lujo para convertirse en una necesidad primaria. El último reporte de la Asociación Colombiana de Vehículos Automotores (ANDEMOS) informa que, hasta Marzo de 2014 se han vendido un total de 68051 automóviles nuevos [1]. Esta cifra sugiere una variación positiva del $7.8 \%$ en relación al mismo periodo de 2013, cuando registraron una caída del 1.9\%; provocando así un aumento en las congestiones vehiculares en las carreteras.

Dicho incremento en las congestiones ha generado un aumento en el tiempo de viaje, consumo de gasolina $\mathrm{y}$ emisiones de Gases de Efecto Invernadero (GEI), causando molestias e incomodidades a los conductores. Por ejemplo, en 2010 un ciudadano norteamericano permanecía en promedio 34 horas y gastaba 14 galones de gasolina por año debido a congestiones vehiculares; comparado con los datos de 1982 (14 horas y 6 galones de gasolina) se observa que el creciente volumen de tráfico es un problema para los sistemas de transporte actuales.

Por lo anterior, las Redes Vehiculares Ad-hoc (VANET: Vehicular Ad-hoc NETworks) presentan alternativas para que los sistemas de transporte actuales no sólo sean más eficientes,

Este proyecto está financiado por el Departamento Administrativo de Ciencia, Tecnología e Innovación (COLCIENCIAS).

978-1-4799-4340-1/14/\$31.00 @ 2014 IEEE sino también más seguros, confiables y convenientes. Estas redes son consideradas como un eje central de los Sistemas Inteligentes de Transporte (ITS: Intelligent Transportation Systems) dada su interoperabilidad con vehículos, semáforos, peatones y demás actores de la vía. También, al no requerir de infraestructura fija y monitorear continuamente el estado de las vías, las VANET son una excelente herramienta para reducir el consumo de gasolina y las emisiones de GEI.

Este artículo se enfatiza en responder la pregunta de cómo aumentar la eficiencia energética de los vehículos en movimiento (disminuir el consumo de gasolina y las emisiones de $\mathrm{CO}_{2}$ ) dependiendo del lugar donde se desplacen. El resultado es el diseño, desarrollo y posterior análisis del desempeño de la aplicación AVO o Aviso de Velocidad Óptima (OSA: Optimal Speed Advisory).

El resto del artículo se organiza de la siguiente manera: la sección II resume el trabajo previo enfocado hacia eficiencia energética. La sección III explica detalles particulares de la aplicación, su lógica y su funcionamiento a nivel físico y nivel MAC, la sección IV describe los parámetros y escenarios de simulación. La sección $\mathrm{V}$ muestra y discute los resultados obtenidos y finalmente la sección VI presenta algunas conclusiones.

\section{TRABAJOS RELACIONADOS}

La investigación en ITS se ha intensificado en los últimos años principalmente porque este campo representa un enorme potencial de mercado e impacto sobre la sociedad. Dentro de los ITS, las VANET pueden mejorar considerablemente la calidad de vida de las personas.

Los autores en [2] investigaron acerca de los efectos de los ITS en el ahorro de energía y la prevención del calentamiento global en Japón, demostrando que las instalaciones actuales en dicho país relacionadas con peajes electrónicos, control de señales de tránsito, ruteo automático y demás contribuyen positivamente a la disminución de emisiones de $\mathrm{CO}_{2}$.

Diversas empresas fabricantes de automóviles han realizado investigaciones enfocadas principalmente a disminuir el impacto ambiental producido por los vehículos. Ford [3], General Motors [4] y BMW [5] se destacan por sus proyectos de sostenibilidad. Además, en Europa el consorcio C2C-CC (Car 2 Car Communication Consortium) es tal vez el más conocido. Fue creado por empresas europeas fabricantes 
de vehículos y actualmente es financiado por productores de repuestos, centros investigativos y otros aliados. Su principal objetivo es "incrementar la seguridad y eficiencia en las vías mediante el uso de Sistemas Inteligentes de Transporte Colaborativos" [6].

En [7] se plantea el caso del transporte inteligente, en donde se analiza que sólo con la construcción de nuevas vías no se terminarán los problemas de congestión e ineficiencia energética, en consecuencia se necesita incrementar la capacidad de las redes de transporte, crear nuevos modelos de negocio y desarrollar sistemas avanzados de control de tráfico para que los ITS solucionen eficazmente estos problemas. Los autores en [8] proponen una metodología para acoplar los estudios que se realicen en distintas ciudades acerca del impacto de los ITS en la movilidad y la sostenibilidad.

La Comisión Europea y el Ministerio de Economía, Industria y Comercio de Japón formaron la fuerza de trabajo EC-METI buscando medir eficazmente el impacto de las aplicaciones de los ITS en las emisiones de $\mathrm{CO}_{2}$ [9], mientras que la ITU presentó un reporte en el cual se define un conjunto de estándares denominado CALM (Continuous Airinterface for Long and Medium range communications), los cuales buscan incrementar el área de cobertura de las redes VANET al brindar mayor soporte para diversas tecnologías como las tres generaciones actuales de telefonía móvil $(2 \mathrm{G}$, $3 \mathrm{G}$, 4G-LTE), redes satelitales, microondas, $\mathrm{WiMAX}^{\circledR} \mathrm{y}$ $\mathrm{Wi}-\mathrm{Fi}{ }^{\circledR}$, entre otras.

Los autores en [10] proporcionan un resumen acerca de las aplicaciones para redes vehiculares desarrolladas y publicadas hasta la fecha, relacionadas principalmente con la disminución de emisiones de $\mathrm{CO}_{2}$, mostrando las principales características, debilidades y retos que presentan los ITS en materia de sostenibilidad ambiental. Además muestran la velocidad óptima promedio a la cual la eficiencia energética es máxima (Ver Fig. 1).

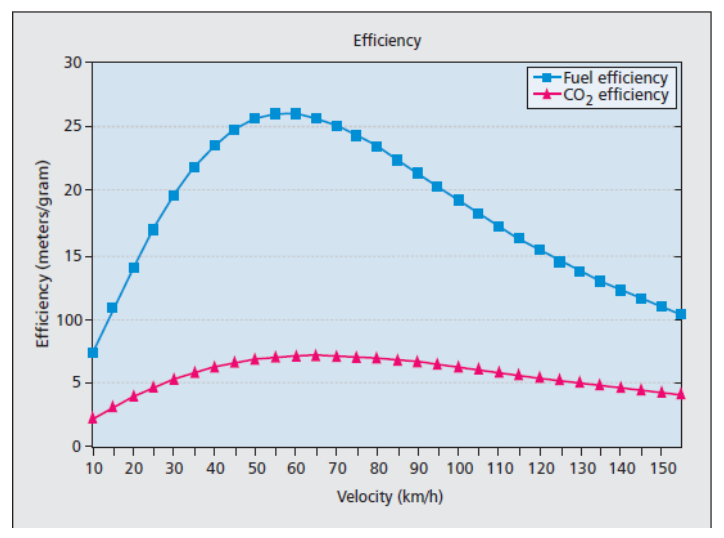

Fig. 1. Eficiencia en motores de gasolina

EcoTrec, un novedoso algoritmo de enrutamiento "ecoamigable" se propone en [11]. Este algoritmo considera características de las vías como las condiciones del pavimento y clima, además de las condiciones actuales del tráfico con el fin de mejorar el ahorro de gasolina y reducir emisiones de GEI. Los autores afirman que utilizando el algoritmo, los vehículos ahorran un $20 \%$ de gasolina en comparación a los automóviles que no lo implementan. En [12], los autores proponen un sistema en tiempo real basado en Comunicaciones Dedicadas de Corto Alcance (DSRC: Dedicated Short Range Communications) buscando reenrutar los vehículos a la ruta más ecológica, evitando carreteras congestionadas y minimizando las emisiones de $\mathrm{CO}_{2}$.

Sommer et al. [13] presenta un estudio comparativo acerca de las emisiones de $\mathrm{CO}_{2}$ contra el tiempo de viaje de los vehículos, analizando la necesidad de considerar múltiples métricas para caracterizar el desempeño de los ITS; entre las cuales están: tiempo de viaje, velocidad promedio, variación de la velocidad, además de las emisiones equivalentes de $\mathrm{CO}_{2}$ por viaje. Los autores concluyen que se debe lograr un equilibrio entre estas variables para que el proceso de optimización en el uso de combustibles sea el más adecuado y se logren reducciones significativas en las emisiones de $\mathrm{CO}_{2}$ a la atmósfera.

Debido a que los trabajos presentados anteriormente están enfocados a escenarios europeos, americanos o japoneses; se requiere de una caracterización al entorno colombiano (y todas sus particularidades) de aplicaciones enfocadas hacia la eficiencia energética. Por lo que la siguiente sección explica detalladamente el diseño de la aplicación propuesta.

\section{DISEÑO DE LA APLICACIÓN}

La aplicación de Aviso de Velocidad Óptima (AVO) tiene como objetivo principal informar a los conductores acerca de la velocidad a la cual las emisiones de $\mathrm{CO}_{2}$ y el consumo de gasolina disminuyen considerablemente sin afectar el tiempo de viaje. La idea principal es que la huella de carbono dejada por los vehículos (nodos) disminuya al optimizar la velocidad con la que circulan por un área específica tomando en cuenta lo estudiado en [10] y mostrado en la Fig. 1.

\section{A. Nivel Físico}

A nivel físico, la aplicación AVO se basa en el estándar IEEE 802.11p que forma parte del conjunto denominado Acceso Inalámbrico en Ambientes Vehiculares (WAVE: Wireless Access in Vehicular Environments). Este toma como base la banda de frecuencias de DSRC de $5.9 \mathrm{GHz}$ y especifica siete canales de $10 \mathrm{MHz}$ cada uno. La Fig. 2 muestra información adicional acerca de dichos canales.

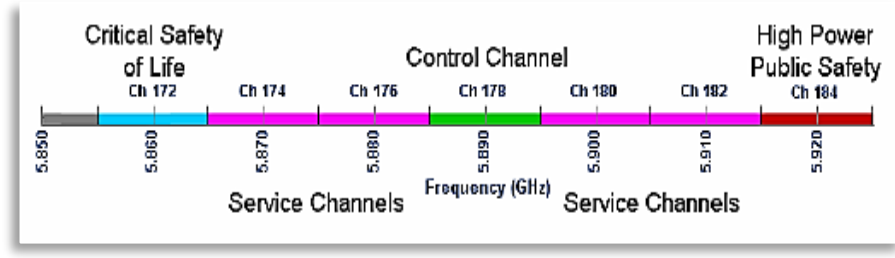

Fig. 2. Canales de DSRC.

El canal utilizado por la aplicación AVO es el canal 184 o HPPS (High Power Public Safety), dado que se requiere que todos los nodos que circulen por la zona de relevancia de la aplicación reciban correctamente los paquetes. 


\section{B. Niveles Superiores}

En los niveles superiores, la aplicación AVO se destaca principalmente por utilizar un enrutamiento de tipo broadcast, dado que se desea establecer comunicación con todos los nodos posibles. Además se utiliza el Protocolo de Mensajes Cortos de WAVE denominado WSMP (WAVE Short Messages Protocol) como protocolo de transporte con el fin de disminuir la latencia de los paquetes.

A nivel de aplicación, AVO recae en la clasificación de Eficiencia de Tráfico ya que optimiza la movilidad de los nodos sobre las carreteras. También es importante señalar que el escenario de comunicación que emplea AVO es el tipo I2V (Infrastructure-to-Vehicle), en donde las RSU (Road Side Units) envían mensajes a los nodos continuamente. La Tabla 1 resume las características de la aplicación.

TABLA I. PARTICULARIDADES DE LA APLICACIÓN AVO

\begin{tabular}{|c|c|}
\hline Característica & AVO \\
\hline Tipo de aplicación & Eficiencia de tráfico \\
\hline Tipo de comunicación & I2V \\
\hline Región de interés & Media \\
\hline Trigger & Periódico \\
\hline Patrón & Uno a muchos \\
\hline Tiempo de vida & $20 \mathrm{~s}$ \\
\hline Frecuencia & $5.92 \mathrm{GHz}$ \\
\hline Canal & HPPS \\
\hline ¿Infraestructura necesaria? & Si \\
\hline Paquete & WSMP \\
\hline Enrutamiento & Broadcast \\
\hline Modo de inicio del protocolo de red & No orientado a conexión \\
\hline Protocolo de transporte &
\end{tabular}

\section{Simuladores y Lógica de la Aplicación}

La simulación en redes vehiculares constituye una herramienta valiosa para analizar y evaluar la viabilidad, los beneficios y las bondades de la implementación de las aplicaciones de estas redes. El grado de realismo y confiabilidad de los resultados de la simulación dependen fundamentalmente de dos aspectos: la integración de un simulador de red con un generador de movilidad y el uso de métricas adecuadas para la evaluación de los resultados [14]. El simulador de red maneja principalmente aspectos relacionados con la comunicación entre vehículos: protocolos, enrutamiento, paquetes y demás; mientras que el generador de movilidad se encarga de modelar lo concerniente al movimiento de los vehículos por las vías: velocidad, aceleración, espacio entre parachoques, etc.

Por otra parte, las tendencias en simulación de redes VANET han evolucionado desde que este tópico empezó a tomar relevancia entre la comunidad científica y académica. En un principio se modeló únicamente el movimiento aleatorio de los nodos con ayuda de la teoría de tráfico, para pasar a tener en cuenta trazas reales, definir escenarios macroscópicos y microscópicos y llegar a la tendencia actual de simulaciones bidireccionalmente acopladas. Este tipo de simulaciones hacen referencia a la comunicación en tiempo real entre el generador de movilidad y el simulador de red, logrando que la eficiencia de simulación aumente considerablemente. La Fig. 3 describe dicha tendencia.

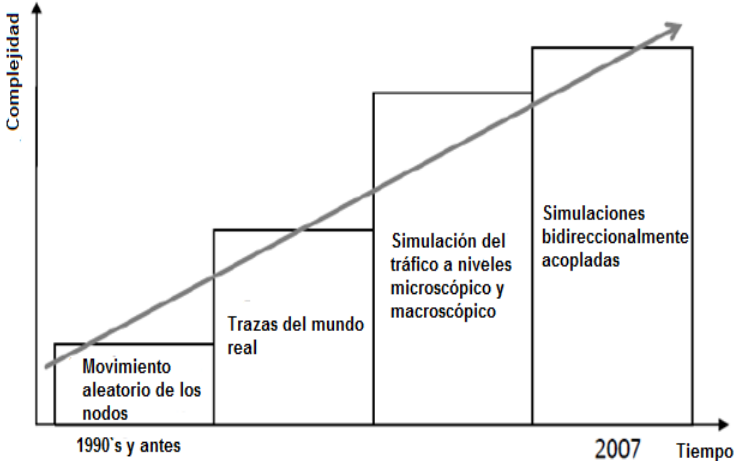

Fig. 3. Tendencias en la simulación de redes VANET

Dado el aumento de las simulaciones bidireccionalmente acopladas han surgido una serie de simuladores de red $\mathrm{y}$ generadores de movilidad que se acoplan entre sí para proveer comunicación en tiempo real. La Tabla II muestra una comparación cualitativa de los simuladores de red más conocidos, mostrando el soporte o no de diversos tópicos; mientras que en la Tabla III se muestra la misma comparación para los generadores de movilidad.

TABLA II. COMPARACIÓN CUALITATIVA DE SIMULADORES DE RED

\begin{tabular}{|c|c|c|c|c|c|c|c|c|}
\hline & NS-2 & GloMoSim & $\begin{array}{c}\text { JiSTI } \\
\text { SWANS }\end{array}$ & QualNet & GTNetS & OMNeT++ & SNS & NS-3 \\
\hline \multicolumn{9}{|l|}{ Software } \\
\hline Portabilidad & $\checkmark$ & $\checkmark$ & $\checkmark$ & $\checkmark$ & $\checkmark$ & $\checkmark$ & $\checkmark$ & $\checkmark$ \\
\hline Freeware & $\checkmark$ & $\checkmark$ & $\checkmark$ & $x$ & $\checkmark$ & $\checkmark$ & $\checkmark$ & $\checkmark$ \\
\hline Open Source & $\checkmark$ & $x$ & $\checkmark$ & $x$ & $\checkmark$ & $\checkmark$ & $\checkmark$ & $\checkmark$ \\
\hline $\begin{array}{l}\text { Ejemplos } \\
\text { Disponibles }\end{array}$ & $\checkmark$ & $\checkmark$ & $\checkmark$ & N.D & $\checkmark$ & $\checkmark$ & $\checkmark$ & $x$ \\
\hline $\begin{array}{l}\text { Desarrollo } \\
\text { continuo }\end{array}$ & $x$ & $x$ & $x$ & $\checkmark$ & $x$ & $\checkmark$ & $x$ & $\checkmark$ \\
\hline $\begin{array}{l}\text { Alto número de } \\
\text { nodos }\end{array}$ & $x$ & $\sigma$ & $\checkmark$ & $\checkmark$ & $x$ & 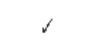 & $\sigma$ & $\checkmark$ \\
\hline $\begin{array}{c}\text { Manejo por } \\
\text { consola }\end{array}$ & $\checkmark$ & $\checkmark$ & $\checkmark$ & N.D & $x$ & $\checkmark$ & $\checkmark$ & $r$ \\
\hline GUI & $\checkmark$ & $\checkmark$ & $\checkmark$ & $\checkmark$ & $\checkmark$ & $\checkmark$ & $x$ & $\checkmark$ \\
\hline Escalabilidad & $x$ & $\checkmark$ & $\checkmark$ & $\checkmark$ & $x$ & $\checkmark$ & $x$ & $\checkmark$ \\
\hline $\begin{array}{l}\text { Facilidad al } \\
\text { configurar }\end{array}$ & $\checkmark$ & $\checkmark$ & $x$ & N.D & $\checkmark$ & $\checkmark$ & $\checkmark$ & $\checkmark$ \\
\hline $\begin{array}{l}\text { Facilidad al utilizar } \\
\text { VANET }\end{array}$ & $x$ & $x$ & $x$ & N.D & $\checkmark$ & $x$ & $x$ & $x$ \\
\hline $802.11 p$ & $\checkmark$ & $x$ & $x$ & $\checkmark$ & $x$ & $\checkmark$ & $x$ & $\checkmark$ \\
\hline Obstáculos & $x$ & $x$ & $x$ & N.D & $x$ & $\checkmark$ & $\checkmark$ & $\checkmark$ \\
\hline $\begin{array}{l}\text { Modelos de flujo y } \\
\text { tráfico vehicular }\end{array}$ & $\hat{x}$ & $\hat{x}$ & $\hat{x}$ & $\gamma$ & $\hat{x}$ & 8 & $x$ & $\gamma$ \\
\hline
\end{tabular}

TABLA III. COMPARACIÓN CUALITATIVA DE GENERADORES DE MOVILIDAD

\begin{tabular}{|c|c|c|c|c|c|c|c|c|c|}
\hline & SUMO & $\begin{array}{l}\text { VanetMobiSim } \\
\end{array}$ & VISSIM & $\begin{array}{l}\text { FreeSim } \\
\end{array}$ & STRAW & MOVE & CityMob & Mobitools & $\begin{array}{l}\text { TraNSlite } \\
\end{array}$ \\
\hline $\begin{array}{l}\text { Solttware } \\
\text { Portabilidad }\end{array}$ & $\checkmark$ & $\checkmark$ & $\gamma$ & $\gamma$ & $\gamma$ & $\checkmark$ & $\checkmark$ & $\gamma$ & $\gamma$ \\
\hline Freeware & $\checkmark$ & $\sigma$ & $\checkmark$ & 8 & $\checkmark$ & $\checkmark$ & $\checkmark$ & $\checkmark$ & $\checkmark$ \\
\hline Opensource & $\sigma$ & $\sigma$ & $\sigma$ & $\sigma$ & $\checkmark$ & $\sigma$ & $\checkmark$ & $x$ & $\mathrm{x}$ \\
\hline Consola de manejo & $\checkmark$ & $\times$ & $\times$ & $\times$ & $\times$ & 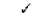 & 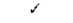 & $\times$ & $\times$ \\
\hline GUI & $\checkmark$ & $\sigma$ & $\checkmark$ & $\times$ & $\checkmark$ & $\checkmark$ & $\sigma$ & $\checkmark$ & $\checkmark$ \\
\hline Ejemplos disponibles & $\checkmark$ & $r$ & $\times$ & $\checkmark$ & $x$ & $\sigma$ & $\times$ & $r$ & $x$ \\
\hline Desarrollo continuo & 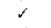 & $\times$ & $x$ & $\times$ & $\times$ & 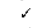 & $x$ & $\times$ & $\times$ \\
\hline Facilidad al configurar & $\checkmark$ & $\checkmark$ & $\sigma$ & $x$ & $\mathrm{x}$ & $\checkmark$ & $\checkmark$ & $\sigma$ & $\sigma$ \\
\hline Facilidad de uso & $\times$ & $\times$ & $\checkmark$ & 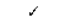 & $\times$ & $\times$ & $\checkmark$ & $\times$ & $\sigma$ \\
\hline \multicolumn{10}{|l|}{ Mapas } \\
\hline Reales & $\checkmark$ & $\sigma$ & $\times$ & 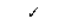 & $\checkmark$ & $\checkmark$ & $x$ & $x$ & $\sigma$ \\
\hline Definidos por el usuario & $\sigma$ & $\sigma$ & 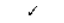 & $\times$ & $\sigma$ & $\checkmark$ & $\times$ & 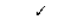 & $\times$ \\
\hline Aleatorios & $\sigma$ & $\sigma$ & $x$ & $\times$ & $\times$ & $\checkmark$ & $\sigma$ & $\checkmark$ & $\checkmark$ \\
\hline Posibilidad de importación & $\checkmark$ & 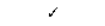 & $\times$ & $\times$ & $\times$ & $\checkmark$ & $\times$ & $\checkmark$ & $\sigma$ \\
\hline \multicolumn{10}{|l|}{ Modelos de movilidad } \\
\hline Mesoscópicos & $\times$ & $\checkmark$ & $\times$ & 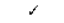 & $\times$ & $\times$ & $\times$ & $\times$ & $\times$ \\
\hline Microscópicos & $\checkmark$ & $\sigma$ & $\sigma$ & $\checkmark$ & $r$ & $\sigma$ & 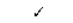 & 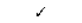 & 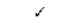 \\
\hline Macroscópicos & $x$ & 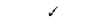 & $x$ & 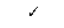 & $x$ & $x$ & $x$ & $x$ & $x$ \\
\hline \multicolumn{10}{|l|}{ Caracteristicas de la via } \\
\hline Vías con múltiples carriles & $\checkmark$ & $\sigma$ & 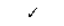 & $x$ & $\sigma$ & $\sigma$ & $\sigma$ & 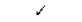 & $\checkmark$ \\
\hline Limitadores de velocidad & 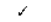 & $\sigma$ & $\times$ & $\sigma$ & $\checkmark$ & $\sigma$ & $\checkmark$ & $\sigma$ & $\checkmark$ \\
\hline Semáforos & $\checkmark$ & $\sigma$ & $\checkmark$ & $x$ & $\sigma$ & $\checkmark$ & $\checkmark$ & $\times$ & 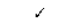 \\
\hline Señales de tránsito & 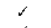 & 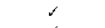 & $x$ & $x$ & $\checkmark$ & $\checkmark$ & $x$ & $\checkmark$ & $\checkmark$ \\
\hline Gestión de intersecciones & 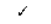 & $\checkmark$ & $\times$ & $\times$ & $\times$ & $\checkmark$ & $\checkmark$ & $\times$ & $\checkmark$ \\
\hline \multicolumn{10}{|l|}{ Trazas } \\
\hline Basadas en XML & 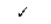 & $\sigma$ & $\times$ & $x$ & $\times$ & $\sigma$ & $x$ & $\times$ & $\times$ \\
\hline Con soporte para NS-2 & $\checkmark$ & $x$ & $x$ & 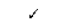 & 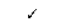 & 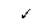 & $\checkmark$ & $x$ & $\checkmark$ \\
\hline Con soporte para Qualnet & $x$ & $\sigma$ & $\times$ & $x$ & $x$ & $\sigma$ & $\times$ & $x$ & $x$ \\
\hline Con soporte para SWANS & $x$ & $x$ & $x$ & $\times$ & & $\times$ & $\times$ & $x$ & $x$ \\
\hline \multicolumn{10}{|l|}{ Otras Caracteristicas } \\
\hline Diferentes tipos de autos & 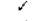 & $\times$ & $\times$ & $\times$ & $\times$ & $\checkmark$ & $\checkmark$ & $\times$ & $\times$ \\
\hline Criterios de adelantamiento & 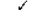 & $x$ & $x$ & $x$ & $x$ & $\checkmark$ & $\times$ & $\times$ & $r$ \\
\hline Cambio de carriles & 5 & (r) & $\times$ & $\times$ & $\times$ & NOVE & cron & 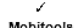 & site \\
\hline
\end{tabular}


Dada la información en las Tablas II y III, los simuladores elegidos para el desarrollo de la aplicación AVO fueron SUMO y OMNeT++, puesto que cumplen con la mayoría de características, entre las cuales se destacan: el soporte para escenarios reales, implementación del protocolo IEEE 802.11p junto con sus estándares IEEE 1609, libertad en licencias (freeware) e interfaces gráficas. Junto a estos dos programas se utilizó el framework denominado Veins (Vehicles in Network Simulation), el cual acopla bidireccionalmente dichas herramientas en tiempo real aumentando la eficiencia computacional de las simulaciones. Veins incorpora diversas librerías para la correcta implementación del conjunto de estándares WAVE, entre las cuales están: Mac1609.4 y sus respectivas interfaces, Decider802.11p, BaseWaveAppLayer, CSMAMacLayer, entre otras. Además, se utilizaron las librerías TraCIMobility y TraCIDemol1p para el movimiento de los nodos.

En OMNeT++ se utilizó un módulo de enrutamiento programado previamente en C [15], capaz de satisfacer las necesidades específicas de AVO y se adecuó el módulo de aplicación. Dichas modificaciones se observan en la Fig. 4

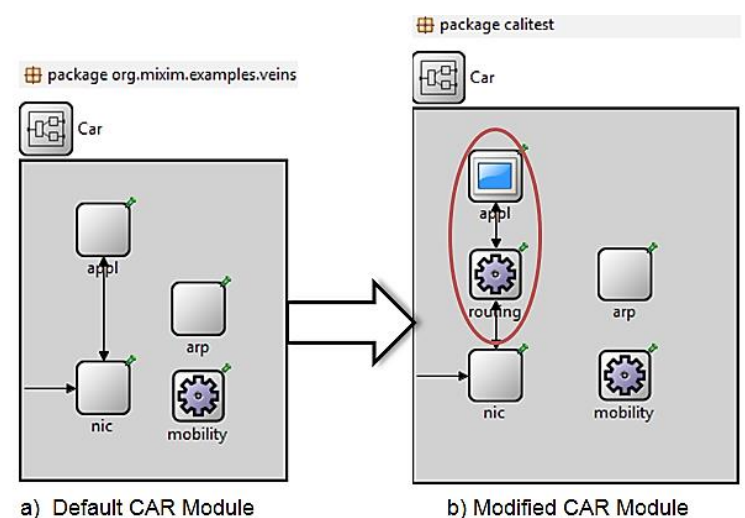

Fig. 4. Modificaciones en OMNeT++

La Fig. 5 muestra la lógica secuencial de la aplicación en donde, si se detecta un nodo circulando por la zona de relevancia, las RSU notifican a los nodos de la velocidad óptima en la zona y almacenan los datos.

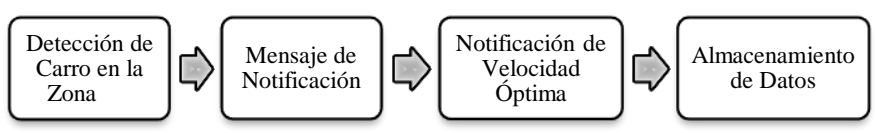

Fig. 5. Lógica de aplicación AVO

El diagrama de eventos para las RSU se muestra en la Fig. 6. De aquí se observa que los mensajes de "Carro en la Zona" y de "Velocidad Óptima Alcanzada" son manejados por SUMO, mientras que el proceso de enrutamiento y transmisión de paquetes es controlado por OMNeT++. Por otra parte, la Fig. 7 describe el diagrama de eventos para los nodos, en donde se presenta el almacenamiento de datos y hay retroalimentación con la red. Es preciso insistir que los mensajes procesados por la aplicación fueron programados con base en el archivo WaveAppMsg.msg (librería de OMNeT++).

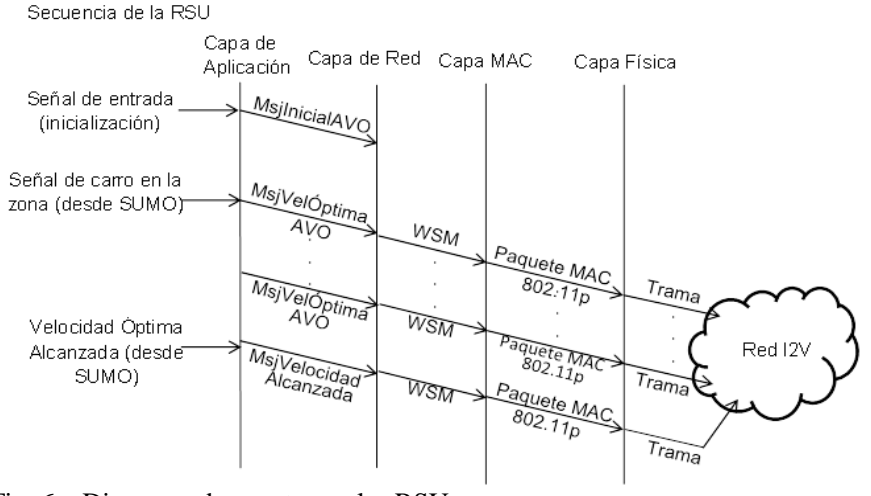

Fig. 6. Diagrama de eventos en las RSU

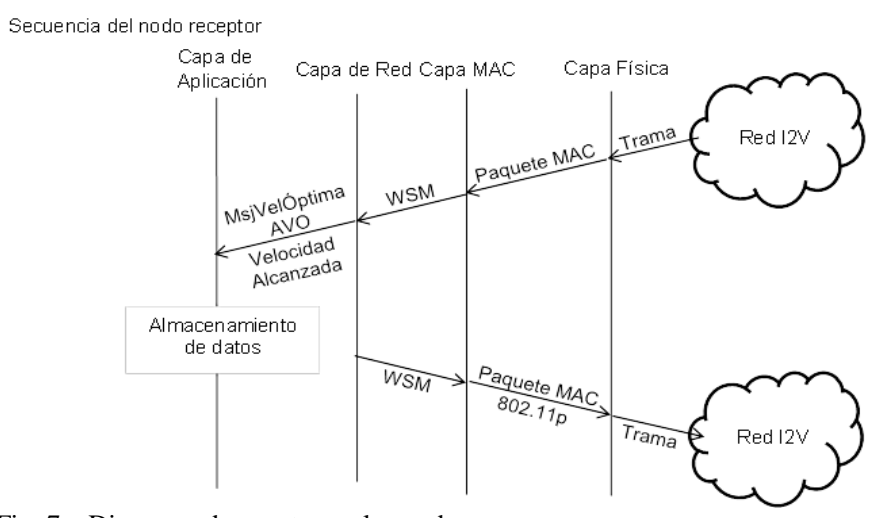

Fig. 7. Diagrama de eventos en los nodos

\section{PARÁMETROS DE SIMULACIÓN}

Para especificar los parámetros de simulación y posteriormente analizar los resultados se siguió la metodología de simulación definida por Ülgen [16], la cual describe:

- Definición de escenarios y casos de simulación.

- Definición de características de los escenarios de simulación.

- Ejecución de las pruebas de simulación.

- Análisis de resultados.

\section{A. Escenarios y Casos de Simulación}

Los escenarios y casos de simulación se definieron de acuerdo al número de nodos que forman parte de la red VANET y del tipo de nodo a simular, pudiendo ser automóviles particulares, vehículos de servicio público, vehículos pesados (camiones) y vans de carga. La Fig. 8 muestra la descripción gráfica de los escenarios y casos.

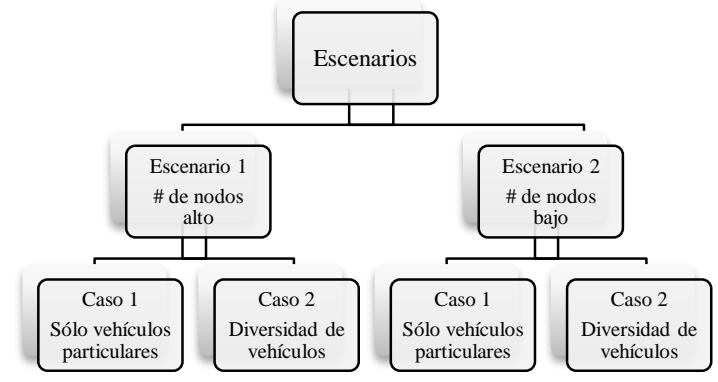

Fig. 8. Escenarios y casos a simular. 
En el escenario 1, el número total de nodos que circulan por la red es de 100, en donde 50 asumen la velocidad óptima que se les informa y la otra mitad ignoran los mensajes informativos. Para el escenario 2, el número de nodos disminuye a 24, y se mantiene la proporción del escenario 1. El caso 1 se destaca por considerar únicamente vehículos particulares, mientras que el caso 2 tiene en cuenta vehículos de transporte público, vehículos pesados y autos particulares.

\section{B. Características de los Escenarios}

Los escenarios descritos anteriormente comparten diversas características entre sí. La descripción de dichas particularidades compartidas se presenta a continuación.

- Área de estudio: para evaluar el desempeño de la aplicación AVO en relación al consumo de gasolina y emisiones de $\mathrm{CO}_{2}$ se requiere de una vía en la que los nodos circulen a altas velocidades y que el flujo de tráfico sea constante. Por esto, se eligió la autopista Simón Bolívar al sur de la ciudad de Cali (Colombia) para cumplir con estos requerimientos. En total los nodos recorren aproximadamente 5 kilómetros en ambos sentidos (sur-norte y norte-sur) y cuatro (4) RSU se ubican al lado de la vía, como se muestra en la Fig. 9.
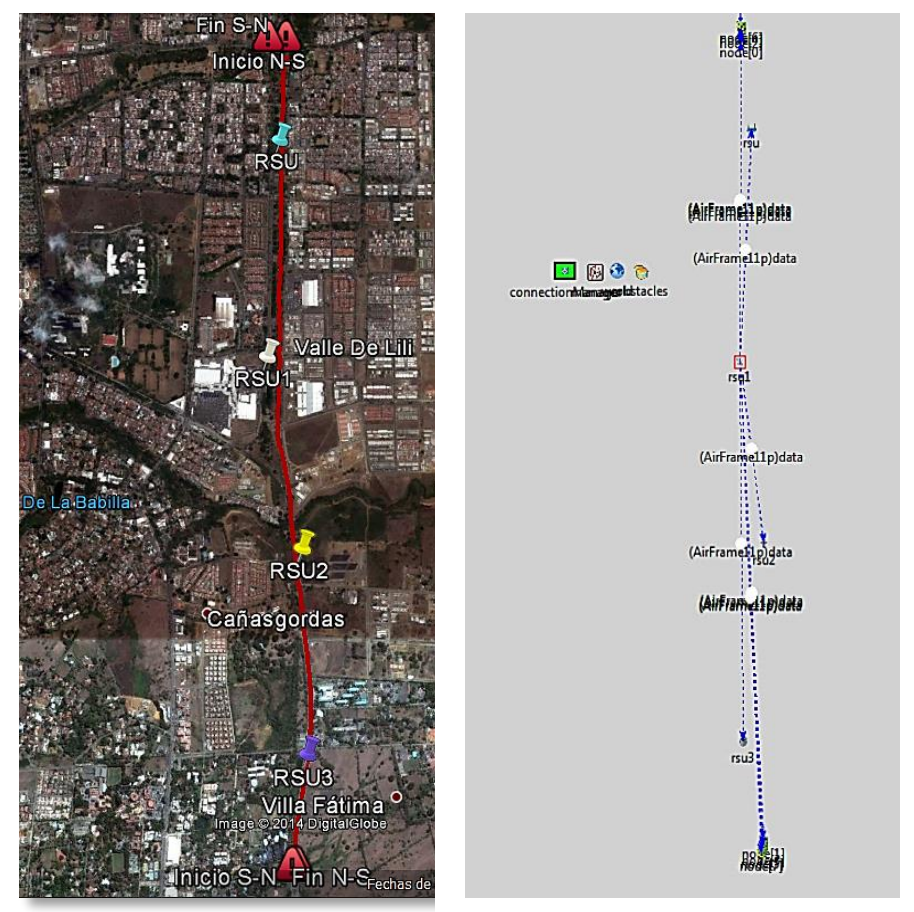

Fig. 9. Área de simulación en Google Earth y en OMNeT++.

- Datos en los Simuladores: en SUMO se implementaron dos archivos que gobiernan la movilidad de los nodos. El tiempo de simulación fue de $\mathbf{6 0 0}$ segundos para el escenario 1 y 450 segundos para el escenario 2. Cada escenario se implementó con muestreo de datos cada 2 segundos y se utilizó el modelo de movilidad IDM (Intelligent Driving Model) para describir el movimiento de los nodos [17]. La Fig. 10 muestra las líneas de código que especifican el modelo de movilidad utilizado y la Tabla 4 resume los datos configurados en los simuladores. 〈routes xmlns:xsi="http://ww, w3,org/2001/XgLSchema-instance" xsi:noNamespaceSche

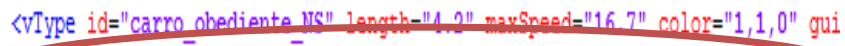
〈CarFollowing-IDM accel="2,00" decel="3.00" timeHeadWay="1" minGap="1" >

Fig. 10. Parámetros de configuración del modelo IDM en SUMO

TABLA IV. CARACTERÍSTICAS EN LOS SIMULADORES

\begin{tabular}{|c|c|c|c|c|}
\hline \multirow{3}{*}{ Característica } & \multicolumn{4}{|c|}{ Ruta y Comportamiento } \\
\hline & \multicolumn{2}{|c|}{ Norte - Sur } & \multicolumn{2}{|c|}{ Sur - Norte } \\
\hline & Acata & No Acata & Acata & No Acata \\
\hline $\begin{array}{l}\text { Velocidad } \\
\text { máxima }\end{array}$ & $\begin{array}{l}16.7 \mathrm{~m} / \mathrm{s} \\
(60 \mathrm{~km} / \mathrm{h})\end{array}$ & $\begin{array}{l}22.2 \mathrm{~m} / \mathrm{s} \\
(80 \mathrm{~km} / \mathrm{h})\end{array}$ & $\begin{array}{c}12.5 \mathrm{~m} / \mathrm{s} \\
(45 \mathrm{~km} / \mathrm{h})\end{array}$ & $\begin{array}{c}18 \mathrm{~m} / \mathrm{s} \\
(65 \mathrm{~km} / \mathrm{h})\end{array}$ \\
\hline $\begin{array}{l}\text { Modelo de } \\
\text { movilidad }\end{array}$ & \multicolumn{4}{|c|}{ IDM } \\
\hline Tipo de conductor & \multicolumn{4}{|c|}{ Agresivo, tímido, camión, normal } \\
\hline Tipo de automóvil & \multicolumn{4}{|c|}{ Carro particular, bus, camión, van de carga } \\
\hline $\begin{array}{l}\text { Potencia de } \\
\text { transmisión }\end{array}$ & \multicolumn{4}{|c|}{$0.8 \mathrm{~W}$} \\
\hline $\begin{array}{l}\text { Umbral de } \\
\text { recepción }\end{array}$ & \multicolumn{4}{|c|}{$-85 \mathrm{dBm}$} \\
\hline Sensibilidad & \multicolumn{4}{|c|}{$-95 \mathrm{dBm}$} \\
\hline Piso de ruido & \multicolumn{4}{|c|}{$-105 \mathrm{dBm}$} \\
\hline $\begin{array}{c}\text { Margen de } \\
\text { desvanecimiento }\end{array}$ & \multicolumn{4}{|c|}{$10 \mathrm{~dB}$} \\
\hline Tasa de datos & \multicolumn{4}{|c|}{$6 \mathrm{Mbps}$} \\
\hline Modulación & \multicolumn{4}{|c|}{ Quadrature Phase Shift Keying (QPSK) } \\
\hline $\begin{array}{c}\text { Tasa de } \\
\text { codificación }\end{array}$ & \multicolumn{4}{|c|}{$1 / 2$} \\
\hline $\begin{array}{l}\text { Modelos de } \\
\text { propagación }\end{array}$ & \multicolumn{4}{|c|}{$\begin{array}{l}\text { Modelo de interferencia de dos rayos; Modelo de } \\
\text { desvanecimiento simple }\end{array}$} \\
\hline
\end{tabular}

\section{Ejecución de Pruebas de Simulación}

La ejecución de las pruebas de simulación se realizó utilizando los simuladores previamente elegidos: SUMO para el manejo del tráfico vehicular, $\mathrm{OMNeT}++$ como plataforma para la implementación de la comunicación de datos y Veins como marco de simulación que los integra. Se realizaron cinco (5) iteraciones por cada escenario y caso estudiado.

\section{Análisis de Resultados}

Tras haber ejecutado las pruebas de simulación correspondientes a los escenarios y casos descritos anteriormente, se obtuvieron resultados relevantes acerca de los siguientes tópicos:

- Gráfica de velocidad óptima vs. paquetes perdidos.

- Gráfica de velocidad no óptima vs. paquetes perdidos.

- Comparación en consumo de gasolina.

- Comparación en emisiones de $\mathrm{CO}_{2}$.

- Gráfica de emisiones de $\mathrm{CO}_{2}$ vs. consumo de gasolina.

- Tasa de Error de Paquetes (PER: Packet Error Rate).

\section{Evaluación DEL DESEMPEÑO}

Para evaluar el desempeño de la aplicación AVO en los escenarios previamente descritos se analizaron una serie de nodos con el fin de observar el consumo de gasolina, emisiones de $\mathrm{CO}_{2}$, pérdida de paquetes y PER. Los datos de emisiones de $\mathrm{CO}_{2}$ son calculados por SUMO con base en el HandBook Emission FActors for road transportation (HBEFA), el cual provee factores de emisión para todas las categorías actuales de vehículos. 


\section{A. Escenario 1}

Se analizaron en total 12 nodos de los 100 que circularon por el escenario de simulación, 6 acatan la velocidad óptima y los restantes 6 la desacatan.

1) Caso 1: en el caso 1, todos los vehículos que circulan son de tipo particular: automóviles normales de cuatro puertas y con las mismas medidas. La Tabla 5 muestra datos relevantes acerca de las emisiones de $\mathrm{CO}_{2}$ en partes por millón (ppm) y gramos, además de la predicción de emisiones anuales de este gas, consumo de gasolina en mililitros (ml) y paquetes procesados por nodo.

De esta tabla se observa que los nodos que circulan en sentido sur-norte presentan, en promedio, un mayor número de paquetes recibidos en relación a los nodos que circulan en sentido norte-sur; esto se da por la velocidad impuesta para cada ruta, en donde la ruta norte-sur impone una mayor velocidad que la ruta sur-norte, de modo que los nodos interaccionan más con las RSU en la ruta sur-norte. Es preciso insistir que las velocidades de las rutas fueron seleccionadas con base a estudios realizados por la Secretaría de Tránsito y Transporte de Cali acerca de la velocidad promedio en la autopista Simón Bolívar (área de estudio) y a los datos de la Fig. 1.

Por otra parte, se observa que los autos que no acatan la velocidad óptima presentan un consumo de gasolina y valor de PER más elevados en relación a los que sí la acatan. Luego, el aumento de velocidad de los nodos incide negativamente en el número de paquetes perdidos. También, al recorrer el área de simulación a la velocidad óptima, las emisiones de $\mathrm{CO}_{2}$ y el consumo de gasolina se reducen en un orden de 500 ppm y 15 $\mathrm{ml}$ respectivamente.

El análisis de las emisiones anuales previstas de $\mathrm{CO}_{2}$ muestra que los nodos que acatan la velocidad óptima dejarían de emitir, en promedio, entre 113.4 y $511.7 \mathrm{~kg}$ de este gas de efecto invernadero a la atmósfera, suponiendo un recorrido anual de $15000 \mathrm{~km}$ por nodo. Este resultado representa una reducción de entre el $5.2 \%$ y el $18.9 \%$ de emisiones de $\mathrm{CO}_{2}$, la cual se adapta a lo propuesto en [18] por el Gobierno Estadounidense como estrategias para la mitigación del cambio climático.

La Fig. 11 muestra la velocidad del nodo 0 y los instantes donde presenta pérdida de paquetes. Durante la primera mitad del recorrido se presenta una mayor pérdida de paquetes; repitiéndose este patrón para los nodos que circulan en sentido norte-sur. Asimismo, la Fig. 12 describe la velocidad del nodo 50 , el cual circula de sur a norte y se observa que la pérdida de paquetes se da al final del trayecto. Esto se da principalmente porque en la parte norte del área de simulación es donde se presenta mayor flujo vehicular y mayor densidad de vehículos; aumentando así el número de paquetes perdidos tanto por colisiones como por un bajo nivel de potencia.

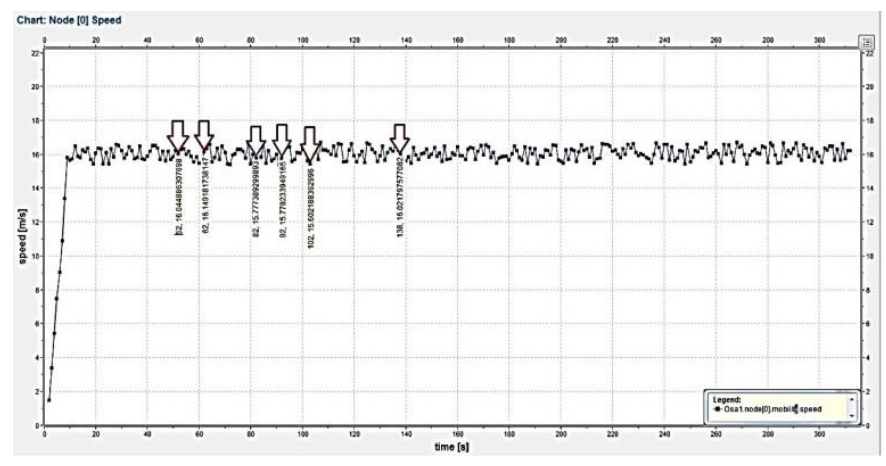

Fig. 11. Velocidad y paquetes perdidos, nodo 0 .

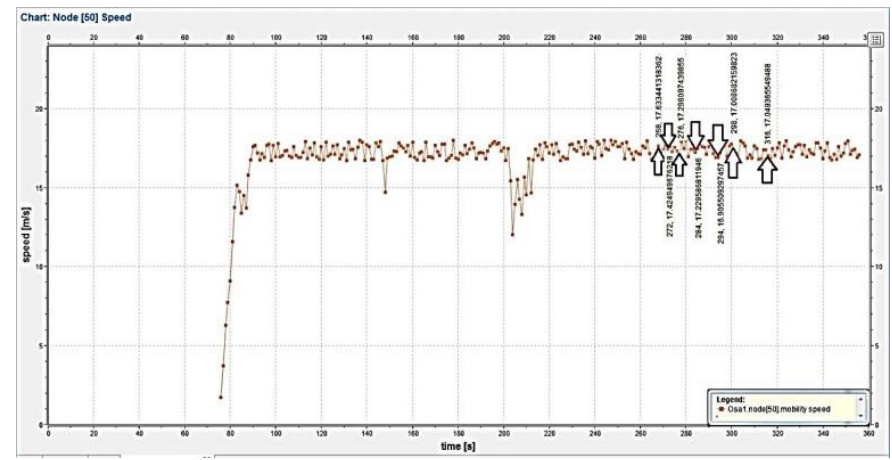

Fig. 12. Velocidad y paquetes perdidos, nodo 50.

2) Caso 2: para este caso se analizaron 6 vehículos particulares, 2 buses, 2 camiones y 2 vans (furgonetas) de carga para observar el impacto que pueda tener la introducción de vehículos pesados a la simulación. La Tabla 6 muestra los datos relevantes acerca de $\mathrm{CO}_{2}, \mathrm{PER}$, paquetes procesados $\mathrm{y}$ gasolina para estos nodos.

Analizando los datos de la Tabla 6 se observa que los vehículos particulares presentan un comportamiento similar en relación al caso 1 (Tabla 5); aunque los vehículos pesados marcan la diferencia en el consumo de gasolina y emisiones de $\mathrm{CO}_{2}$. Los valores de PER y el consumo de gasolina continúan siendo menores para los nodos que acatan la velocidad, comprobando así la eficiencia de AVO.

En los vehículos pesados se observa un incremento considerable en las emisiones de $\mathrm{CO}_{2}$ y consumo de gasolina, debido al peso de éstos autos, al tipo de motor y combustible utilizado. Las emisiones anuales de $\mathrm{CO}_{2}$ esperadas se disparan hasta las 25 toneladas para los camiones y entre 8 y 10 toneladas para buses de transporte público.

Los valores de PER para los nodos que no acatan la velocidad óptima son mayores en relación a los mostrados en la tabla 5, ya que se presenta una mayor congestión debido a los tamaños de los vehículos pesados. Por otra parte, de los datos de ambas tablas se observa una relación directa entre emisiones de $\mathrm{CO}_{2}$ y consumo de gasolina; esta relación se muestra gráficamente en la Fig. 13 para un nodo en particular, pero todos los nodos siguieron dicha tendencia en la que a mayor emisión de $\mathrm{CO}_{2}$, mayor consumo de gasolina. 
TABLA V. RESUMEN DE PAQUETES PROCESADOS POR NODO, $\mathrm{CO}_{2}$ EMITIDO Y CONSUMO DE GASOLINA, EsCENARIO 1 CASO 1

\begin{tabular}{|c|c|c|c|c|c|c|c|c|c|}
\hline Nodo & Ruta & $\begin{array}{c}\text { iAcata la } \\
\text { velocidad? }\end{array}$ & $\begin{array}{c}\text { Paquetes } \\
\text { recibidos }\end{array}$ & $\begin{array}{c}\text { Paquetes } \\
\text { perdidos- } \\
\text { colisionados }\end{array}$ & PER & $\begin{array}{c}\mathbf{C O}_{2} \text { promedio } \\
\text { emitido (ppm) }\end{array}$ & $\begin{array}{c}\mathbf{C O}_{2} \text { total } \\
\text { emitido (g) }\end{array}$ & $\begin{array}{c}\text { Consumo } \\
\text { de gasolina } \\
\text { (ml) }\end{array}$ & $\begin{array}{c}\text { Emisiones } \\
\text { anuales de } \\
\text { CO }\end{array}$ (kg) \\
\hline 0 & Norte-Sur & $\mathrm{Si}$ & 97 & $8-6$ & $76 \times 10^{-3}$ & 1498 & 841.3 & 304.2 & 2523.9 \\
\hline 28 & Norte-Sur & $\mathrm{Si}$ & 90 & $6-5$ & $63 \times 10^{-3}$ & 1513 & 850.0 & 313.7 & 2550.0 \\
\hline 49 & Norte-Sur & $\mathrm{Si}$ & 91 & $7-6$ & $71 \times 10^{-3}$ & 1505 & 849.8 & 313.2 & 2549.4 \\
\hline 2 & Norte-Sur & $\mathrm{No}$ & 68 & $10-7$ & $128 \times 10^{-3}$ & 2068 & 899.6 & 325.0 & 2698.8 \\
\hline 22 & Norte-Sur & $\mathrm{No}$ & 80 & $11-6$ & $121 \times 10^{-3}$ & 1996 & 884.5 & 323.2 & 2710.4 \\
\hline 43 & Norte-Sur & $\mathrm{No}$ & 69 & $12-8$ & $148 \times 10^{-3}$ & 2014 & 887.8 & 324.1 & 2663.4 \\
\hline 57 & Sur-Norte & $\mathrm{Si}$ & 124 & $4-3$ & $31 \times 10^{-3}$ & 1196 & 741.2 & 294.6 & 2223.6 \\
\hline 69 & Sur-Norte & $\mathrm{Si}$ & 127 & $8-6$ & $59 \times 10^{-3}$ & 1175 & 732.9 & 294.1 & 2198.7 \\
\hline 88 & Sur-Norte & $\mathrm{Si}$ & 120 & $7-5$ & $55 \times 10^{-3}$ & 1200 & 744.3 & 297.5 & 2232.9 \\
\hline 50 & Sur-Norte & $\mathrm{No}$ & 82 & $10-7$ & $109 \times 10^{-3}$ & 1701 & 865.8 & 319.0 & 2597.4 \\
\hline 78 & Sur-Norte & $\mathrm{No}$ & 85 & $12-9$ & $124 \times 10^{-3}$ & 1683 & 872.1 & 318.1 & 2616.3 \\
\hline 98 & Sur-Norte & No & 87 & $14-11$ & $139 \times 10^{-3}$ & 1696 & 865.7 & 318.9 & 2597.1 \\
\hline
\end{tabular}

TABLA VI. Resumen de Paquetes Procesados POR NOdo, $\mathrm{CO}_{2}$ EMItido y CONSUMo de Gasolina, Escenario 1 CASO 2

\begin{tabular}{|c|c|c|c|c|c|c|c|c|c|c|}
\hline Nodo & $\begin{array}{c}\text { Tipo de } \\
\text { vehículo }\end{array}$ & Ruta & $\begin{array}{c}\text { ¿Acata la } \\
\text { velocidad? }\end{array}$ & $\begin{array}{l}\text { Paquetes } \\
\text { recibidos }\end{array}$ & $\begin{array}{c}\text { Paquetes } \\
\text { perdidos- } \\
\text { colisionados }\end{array}$ & PER & $\begin{array}{l}\mathrm{CO}_{2} \text { promedio } \\
\text { emitido (ppm) }\end{array}$ & $\begin{array}{c}\mathrm{CO}_{2} \text { total } \\
\text { emitido }(\mathrm{g})\end{array}$ & $\begin{array}{c}\text { Consumo } \\
\text { de gasolina } \\
(\mathrm{ml})\end{array}$ & $\begin{array}{c}\text { Emisiones } \\
\text { anuales de } \\
\mathrm{CO}_{2}(\mathrm{~kg})\end{array}$ \\
\hline 0 & Particular & Norte-Sur & $\mathrm{Si}$ & 113 & $3-3$ & $26 \times 10^{-3}$ & 1516 & 848.9 & 308.8 & 2546.7 \\
\hline 30 & Van & Norte-Sur & $\mathrm{Si}$ & 95 & $6-4$ & $59 \times 10^{-3}$ & 1655 & 904.4 & 332.3 & $4522.0^{\mathrm{b}}$ \\
\hline 11 & Camión & Norte-Sur & No & 101 & $22-16$ & $179 \times 10^{-3}$ & 8695 & 4883.1 & 1881.5 & $24415.5^{b}$ \\
\hline 32 & Particular & Norte-Sur & No & 81 & $15-10$ & $156 \times 10^{-3}$ & 2031 & 892.9 & 325.5 & 2678.7 \\
\hline 48 & Camión & Norte-Sur & No & 87 & $19-13$ & $179 \times 10^{-3}$ & 9122 & 5122.9 & 1981.5 & $25614.5^{b}$ \\
\hline 89 & Bus & Sur-Norte & $\mathrm{Si}$ & 117 & $11-9$ & $86 \times 10^{-3}$ & 2977 & 2124.4 & 746.4 & $8497.6^{\mathrm{b}}$ \\
\hline 51 & Particular & Sur-Norte & No & 96 & $17-7$ & $150 \times 10^{-3}$ & 1890 & 904.5 & 329.0 & 2713.5 \\
\hline 82 & Van & Sur-Norte & No & 84 & $14-10$ & $143 \times 10^{-3}$ & 2026 & 1104.1 & 355.1 & $5520.5^{\mathrm{b}}$ \\
\hline 92 & Particular & Sur-Norte & No & 68 & $13-9$ & $160 \times 10^{-3}$ & 1837 & 897.7 & 328.2 & 2693.1 \\
\hline
\end{tabular}

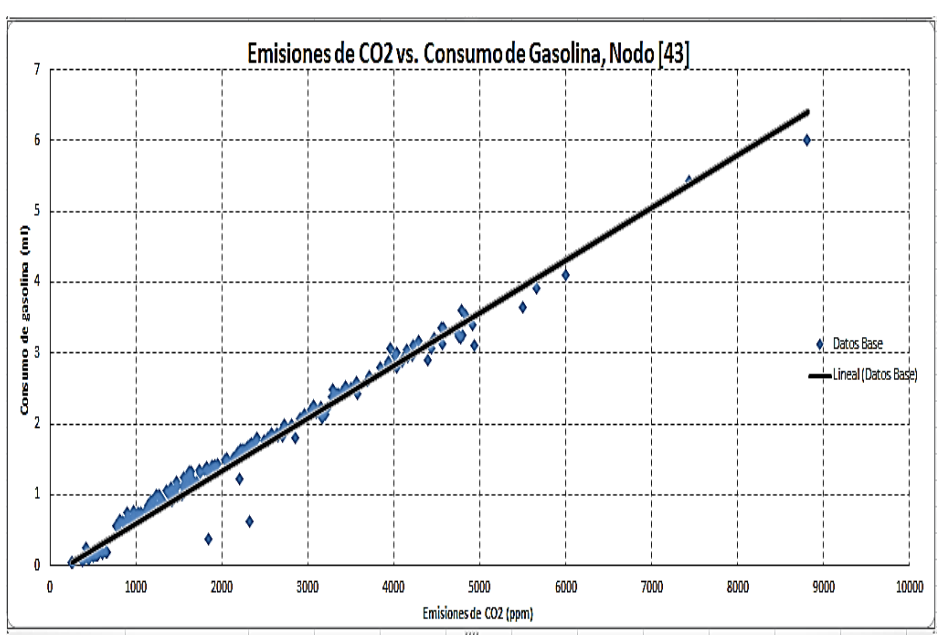

Fig. 13. Relación entre consumo de gasolina y emisiones de CO2.

\section{B. Escenario 2}

En este escenario circulan un total de 24 nodos, de los cuales 12 acatan la velocidad óptima y 12 hacen caso omiso. Al igual que el escenario 1 , se analizaron 12 nodos.

1) Caso 1: la Tabla 7 describe el resumen de paquetes, $\mathrm{CO}_{2}$ y consumo de gasolina para el caso en el que todos los nodos que circulan por el área de simulación son automóviles particulares. De esta tabla se observa que el número de paquetes perdidos por colisiones es menor en comparación al escenario 1 para todos los nodos; principalmente porque hay menos vehículos circulando por la vía y la posibilidad de que haya colisiones de paquetes disminuye considerablemente. Continúa la tendencia de que los nodos que acatan la velocidad óptima presentan mejor eficiencia en el procesamiento de los paquetes, consumo de gasolina $\mathrm{y}$ emisiones de $\mathrm{CO}_{2}$; además las emisiones anuales esperadas de este gas por parte de los nodos que acatan la velocidad óptima disminuyen entre el $3.2 \%$ y el $17.4 \%$ por cada $15000 \mathrm{~km}$ recorridos.

2) Caso 2: la Tabla 8 muestra los datos relevantes en relación al consumo de $\mathrm{CO}_{2}$, PER y consumo de gasolina cuando se tienen en cuenta diversos tipos de vehículos. En esta tabla se observa que, al igual que el caso anterior, el número de paquetes perdidos por colisión es menor al escenario 1 y los nodos que acatan la velocidad óptima siguen presentando un menor valor de PER y mejor eficiencia energética. El porcentaje de ahorro de emisiones anuales de $\mathrm{CO}_{2}$ varía entre el $5.3 \%$ y el $11.8 \%$ para vehículos particulares y el ahorro para vans se ubica en el $26.6 \%$. Esto indica que, para vehículos pesados, la aplicación AVO es más eficiente y ayuda a disminuir en mayor grado las emisiones anuales de $\mathrm{CO}_{2}$.

a. El cálculo anual de emisiones de $\mathrm{CO}_{2}$ relaciona la cantidad de este gas en gramos con la distancia en estudio y el kilometraje promedio por año así [19]:

$$
\left(X^{g} / 5 \mathrm{~km}\right) \times(15000 \mathrm{~km} / 1 \text { año }) \times\left(1^{\mathrm{kg}} / 1000 \mathrm{~g}\right)
$$

b. Para el caso de los vehículos pesados se tomó en cuenta lo descrito en $[19,20]$ en donde se sugiere un kilometraje por año promedio para vans de 25000 km/año; para buses urbanos de $20000 \mathrm{~km} / \mathrm{año} \mathrm{y} \mathrm{para} \mathrm{camiones} \mathrm{de} 25000 \mathrm{~km} / \mathrm{año}$. 
TABLA VII. RESUMEN DE PAQUETES PROCESADOS POR NODO, $\mathrm{CO}_{2}$ EMITIDO Y CONSUMO DE GASOLINA, EsCENARIO 2 CASO 1

\begin{tabular}{|c|c|c|c|c|c|c|c|c|c|}
\hline Nodo & Ruta & $\begin{array}{c}\text { iAcata la } \\
\text { velocidad? }\end{array}$ & $\begin{array}{c}\text { Paquetes } \\
\text { recibidos }\end{array}$ & $\begin{array}{c}\text { Paquetes } \\
\text { perdidos- } \\
\text { colisionados }\end{array}$ & PER & $\begin{array}{c}\mathbf{C O}_{2} \text { promedio } \\
\text { emitido (ppm) }\end{array}$ & $\begin{array}{c}\mathbf{C O}_{2} \text { total } \\
\text { emitido (g) }\end{array}$ & $\begin{array}{c}\text { Consumo } \\
\text { de gasolina } \\
\text { (ml) }\end{array}$ & $\begin{array}{c}\text { Emisiones } \\
\text { anuales de } \\
\text { CO }\end{array}$ (kg) \\
\hline 0 & Norte-Sur & $\mathrm{Si}$ & 103 & $8-3$ & $73 \times 10^{-3}$ & 1522 & 855.0 & 308.3 & 2565.0 \\
\hline 8 & Norte-Sur & $\mathrm{Si}$ & 103 & $8-2$ & $73 \times 10^{-3}$ & 1502 & 848.7 & 306.6 & 2546.1 \\
\hline 20 & Norte-Sur & $\mathrm{Si}$ & 103 & $8-3$ & $73 \times 10^{-3}$ & 1472 & 832.0 & 300.1 & 2496.0 \\
\hline 2 & Norte-Sur & $\mathrm{No}$ & 72 & $10-3$ & $122 \times 10^{-3}$ & 1999 & 894.9 & 329.9 & 2684.7 \\
\hline 10 & Norte-Sur & $\mathrm{No}$ & 63 & $9-4$ & $127 \times 10^{-3}$ & 2066 & 899.3 & 331.7 & 2697.9 \\
\hline 22 & Norte-Sur & $\mathrm{No}$ & 68 & $10-4$ & $128 \times 10^{-3}$ & 2010 & 899.1 & 331.5 & 2697.3 \\
\hline 5 & Sur-Norte & $\mathrm{Si}$ & 125 & $12-5$ & $88 \times 10^{-3}$ & 1217 & 789.3 & 297.0 & 2367.9 \\
\hline 13 & Sur-Norte & $\mathrm{Si}$ & 121 & $9-3$ & $69 \times 10^{-3}$ & 1209 & 785.4 & 296.1 & 2356.2 \\
\hline 17 & Sur-Norte & $\mathrm{Si}$ & 118 & $10-4$ & $78 \times 10^{-3}$ & 1112 & 749.0 & 290.4 & 2247.0 \\
\hline 7 & Sur-Norte & $\mathrm{No}$ & 98 & $15-6$ & $133 \times 10^{-3}$ & 1725 & 887.7 & 326.8 & 2663.1 \\
\hline 15 & Sur-Norte & $\mathrm{No}$ & 91 & $14-5$ & $133 \times 10^{-3}$ & 1712 & 882.1 & 325.5 & 2646.3 \\
\hline 19 & Sur-Norte & No & 82 & $17-7$ & $171 \times 10^{-3}$ & 1781 & 906.5 & 335.5 & 2719.5 \\
\hline
\end{tabular}

TABLA VIII. Resumen de Paquetes Procesados POR NOdo, $\mathrm{CO}_{2}$ EMITIDO Y CONSUMO de Gasolina, EsCENARIO 2 CASO 2

\begin{tabular}{|c|c|c|c|c|c|c|c|c|c|c|}
\hline Nodo & $\begin{array}{c}\text { Tipo de } \\
\text { vehículo }\end{array}$ & Ruta & $\begin{array}{c}\text { ¿Acata la } \\
\text { velocidad? }\end{array}$ & $\begin{array}{l}\text { Paquetes } \\
\text { recibidos }\end{array}$ & $\begin{array}{c}\text { Paquetes } \\
\text { perdidos- } \\
\text { colisionados }\end{array}$ & PER & $\begin{array}{l}\mathrm{CO}_{2} \text { promedio } \\
\text { emitido (ppm) }\end{array}$ & $\begin{array}{c}\mathrm{CO}_{2} \text { total } \\
\text { emitido }(\mathrm{g})\end{array}$ & $\begin{array}{c}\text { Consumo } \\
\text { de gasolina } \\
(\mathrm{ml})\end{array}$ & $\begin{array}{c}\text { Emisiones } \\
\text { anuales de } \\
\mathrm{CO}_{2}(\mathrm{~kg})\end{array}$ \\
\hline 0 & Particular & Norte-Sur & $\mathrm{Si}$ & 82 & $6-1$ & $68 \times 10^{-3}$ & 1522 & 857.4 & 310.1 & 2572.2 \\
\hline 14 & Van & Norte-Sur & $\mathrm{Si}$ & 93 & $9-3$ & $88 \times 10^{-3}$ & 1688 & 951.0 & 366.3 & 4755.0 \\
\hline 8 & Camión & Norte-Sur & No & 101 & $15-4$ & $129 \times 10^{-3}$ & 8827 & 4957.3 & 1913.4 & 24786.5 \\
\hline 18 & Particular & Norte-Sur & No & 79 & $12-2$ & $132 \times 10^{-3}$ & 2015 & 899.6 & 331.5 & 2698.8 \\
\hline 22 & Camión & Norte-Sur & No & 104 & $17-6$ & $141 \times 10^{-3}$ & 8575 & 4831.0 & 1876.9 & 24155.0 \\
\hline 23 & Bus & Sur-Norte & $\mathrm{Si}$ & 129 & $11-6$ & $78 \times 10^{-3}$ & 3447 & 2512.5 & 944.1 & 10050.0 \\
\hline 16 & Particular & Sur-Norte & No & 83 & $12-4$ & $125 \times 10^{-3}$ & 1771 & 892.1 & 330.0 & 2676.3 \\
\hline 21 & Van & Sur-Norte & No & 75 & $13-5$ & $148 \times 10^{-3}$ & 2043 & 1296.3 & 413.4 & 6481.5 \\
\hline 11 & Particular & Sur-Norte & No & 82 & $13-6$ & $137 \times 10^{-3}$ & 1793 & 896.3 & 333.3 & 2688.9 \\
\hline
\end{tabular}

\section{CONCLUSIONES}

En este trabajo de investigación se diseñó y desarrolló una aplicación para redes vehiculares que optimiza la eficiencia energética y el impacto ambiental de los vehículos al informar de la velocidad óptima en un área dada. Combina las ventajas de las redes VANET en términos de portabilidad y escalabilidad para reducir las emisiones de $\mathrm{CO}_{2}$ y el consumo de gasolina. Los resultados de simulación verifican los beneficios de emplear esta aplicación y de acatar la información enviada por las RSU: las emisiones de $\mathrm{CO}_{2}$ y el consumo de gasolina disminuyen considerablemente para todos los nodos que acataron la información recibida; mientras que los nodos que ignoran dicha información presentan un consumo más elevado de gasolina y mayores emisiones anuales de este gas. En cada escenario y caso considerado, la aplicación demostró fiabilidad y robustez al disminuir el valor de PER y de paquetes perdidos para los nodos que acatan su información. Esta mejoría en la eficiencia energética se presentó para todos los tipos de vehículos simulados, ya sean automóviles particulares, buses, vans de carga o camiones.

\section{RECONOCIMIENTO}

Esta investigación es financiada por el Departamento Administrativo de Ciencia, Tecnología e Innovación (COLCIENCIAS), entidad gubernamental Colombiana y su programa de Jóvenes Investigadores.

\section{REFERENCIAS}

[1] Asociación Colombiana de Vehículos Automotores, ANDEMOS, Estadísticas Sector Automotor, Marzo de 2014 [Online], disponible en: http://www.andemos.org/categoria.php?c=12\&sub=18, consultado: [24 Abr 2014].

[2] S. Tsugawa y S. Kato, "Energy ITS: Another Application of Vehicular Communications", IEEE Communications Magazine, vol.48, no.11, pp.120-126, Noviembre 2010.

[3] W.C. Ford, Jr y A. Mulally, "Sustainability Report Summary 2012/13," Ford white paper, [Online], disponible en: http://corporate.ford.com/doc/sr12-summary.pdf, consultado: [22 Ene 2014].

[4] General Motors Collaborative Research Lab, Electrical \& Computer Engineering, Carnegie Mellon University, [Online], disponible en: http://gm.web.cmu.edu/, consultado: [23 Ene 2014].

[5] K. Draeger, PhD, "BMW Group Research and Technology. Creative Power - customer oriented and efficient," white paper, [Online], disponible

http://www.bmwgroup.com/e/0_0_www_bmwgroup_com/unternehmen/ publikationen/aktuelles_lexikon/_pdf/alex_forschung_technik_10_05_fi nal.pdf, consultado: [23 Ene 2014].

[6] Car 2 Car Communication Consortium Manifesto, Overview of the C2C-CC System, Agosto 2007.

[7] The Case for Smart Transportation, International Business Machines Sales and Distribution Whitepaper, IBM Corporation, Septiembre 2010.

[8] T. Mehta, A. Kottapalli, H. S. Mahmassani y C. Bhat, "Intelligent Transportation Systems and the Environment," Reporte Técnico 4197-S, Proyecto O-4197, Centro para la Investigación en Transporte, Universidad de Texas en Austin, Enero 2010. 
[9] European Commision \& Ministry of Economy, Trade and Industry(ECMETI), Methodologies for Assessing the Impact of ITS Applications on $\mathrm{CO}_{2}$ Emissions, Technical Report V1.0, Marzo 2009.

[10] C. Suthaputchakun, Z. Sun y M. Dianati, "Applications of Vehicular Communications for Reducing Fuel Consumption and $\mathrm{CO} 2$ Emission: The State of the Art and Research Challenges", IEEE Communications Magazine, vol.50, no.12, pp.108-115, Diciembre 2012.

[11] R. Doolan y G. Muntean, "VANET-enabled Eco-friendly Road Characteristics-aware Routing for Vehicular Traffic," en $77^{\text {th }}$ IEEE Vehicular Technology Conference, Dresden, Alemania, Junio 2013, pp. $1-5$.

[12] L. Maglaras, P. Basaras y D, Katsaros, "Exploiting vehicular communications for reducing CO2 emissions in urban environments," en Internat. Conf. on Connected Vehicles and Expo, ICCVE, Las Vegas, Nevada: USA, Diciembre 2013, pp. 32-37.

[13] C. Sommer et al., "Emissions vs. Travel Time: Simulative Evaluation of the Environmental Impact of ITS," IEEE $71^{\text {st }}$ Vehicular Technology Conference, Taipei: Taiwan, Mayo 2010, pp. 1-5.

[14] F. Dressler et al., "Towards Realistic Simulation of Inter-Vehicle Communication: Models, Techniques and Pitfalls," IEEE Vehicular Technology Magazine, vol. 6, pp. 43-51, Septiembre 2011.

[15] A. Orozco, R. Michoud y G. Llano, "Efficiency Applications for Vehicular Networks: Towards Green Transportation Systems," en IEEE Latin-American Conference on Communications (LATINCOM), Cuenca: Ecuador, Noviembre 2012.

[16] O. Ülgen, Simulation Methodology - A Practitioner's Perspective. Dearborn, Michigan: USA, University of Michigan-Dearborn, 2006.

[17] A. Kesting, M. Treiber y D. Helbing, Agents for Traffic Simulation, Multi-Agent Systems: Simulation and Applications, Cap. 11, CRC Press, Mayo 2008 pp. 325-356.

[18] United States Department of State. U.S. Climate Action Report 2010. Washington: Golbal Publishing Services, Junio 2010.

[19] United States Environmental Protection Agency (EPA), Office of Transportation and Air Quality, Average Annual Emissions and Fuel Consumption for Gasoline-Fueled Passenger Cars and Light Trucks, Reporte Técnico EPA420-F-08-024, Octubre 2008.

[20] R. Moller, D. Beltrán y F. Jiménez, La Alternativa para el Transporte Público Colectivo en Colombia, Programa Editorial Universidad del Valle, Cali, Colombia, 2004. 